CARPATHIAN J. MATH.

Volume 38 (2022), No. 1,

Pages 129 - 137
Online version at https : //www . carpathian. cunbm. utcluj. ro/

Print Edition: ISSN 1584 - 2851; Online Edition: ISSN 1843 - 4401

DOI: https://doi.org/10.37193/CJM.2022.01.10

Dedicated to the memory of Academician Mitrofan M. Choban (1942-2021)

\title{
Darboux integrability of a cubic differential system with two parallel invariant straight lines
}

\author{
DUMitru COZMA
}

ABSTRACT. In this paper we prove the Darboux integrability of a cubic differential system with a singular point of a center typer having at least two parallel invariant straight lines.

\section{INTRODUCTION}

We consider the real cubic differential system

$$
\dot{x}=P(x, y), \quad \dot{y}=Q(x, y),
$$

where $P(x, y)$ and $Q(x, y)$ are coprime polynomials in $\mathbb{R}[x, y]$. Let the origin $O(0,0)$ be an isolated singularity of (1.1) with purely imaginary eigenvalues $\left(\lambda_{1,2}= \pm i, i^{2}=-1\right)$. Then by using a nondegenerate transformation of variables and a time rescaling, the system (1.1) can be brought to the form

$$
\begin{aligned}
& \dot{x}=y+a x^{2}+c x y+f y^{2}+k x^{3}+m x^{2} y+p x y^{2}+r y^{3} \equiv P(x, y), \\
& \dot{y}=-\left(x+g x^{2}+d x y+b y^{2}+s x^{3}+q x^{2} y+n x y^{2}+l y^{3}\right) \equiv Q(x, y),
\end{aligned}
$$

where the variables and coefficients are assumed to be real. The trajectories in some neighborhood of $O(0,0)$ can be spirals or closed trajectories. Thus, the origin is either a focus or a center for system (1.2), i.e. a fine focus.

The problem of distinguishing between a center and a focus (the problem of the center) is open for general cubic differential systems. An approach to the problem of the center for cubic differential system (1.2) is to study the local integrability of the system in some neighborhood of the singular point $O(0,0)$.

Using the method of Darboux integrability and the rational reversibility, the problem of the center was solved for cubic system (1.2) with: four invariant straight lines [5]; three invariant straight lines [7], [17]; two invariant straight lines and one irreducible invariant conic [6]; two invariant straight lines and one irreducible invariant cubic [10]. The center conditions for a cubic system (1.2) with two distinct invariant straight lines by using the method of Darboux integrability and rational reversibility were found in [8].

The Darboux integrability conditions were obtained for some reversible cubic differential systems (1.2) in [2] and for a few families of the complex cubic system in [11].

In this paper we study the problem of integrability for cubic differential system (1.2) with two parallel invariant straight lines. We obtain fourteen families of cubic systems with at least two parallel invariant straight lines which are Darboux integrable.

Received: 28.09.2021. In revised form: 04.11.2021. Accepted: 11.11.2021

2010 Mathematics Subject Classification. 34C05.

Key words and phrases. Cubic differential systems, the problem of the center, invariant algebraic curves, Darboux integrability. 


\section{INVARIANT ALGEBRAIC CURVES}

We are interested in the algebraic integrability of cubic differential systems which have a given set of irreducible invariant algebraic curves, called algebraic solutions.

Definition 2.1. An algebraic invariant curve (or an algebraic particular integral) of (1.2) is the solution set in $\mathbb{C}^{2}$ of an equation $\Phi(x, y)=0$, where $\Phi$ is a polynomial in $x, y$ with complex coefficients such that

$$
\frac{\partial \Phi}{\partial x} P(x, y)+\frac{\partial \Phi}{\partial y} Q(x, y)=\Phi K,
$$

for some polynomial in $x, y, K=K(x, y)$ with complex coefficients, called the cofactor of the invariant algebraic curve $\Phi(x, y)=0$.

It is a very hard problem to calculate the invariant algebraic curves for a given differential system of degree $n$ because, in general, we do not have any evidence on the number of invariant algebraic curves and on the degree of a curve [13].

Open problem 1. What is the maximum number of algebraic invariant curves in the set of all polynomial differential systems of degree $n>1$ having finitely many invariant algebraic curves?

Open problem 2. Give a method to find an upper bound to the degree of the algebraic solutions for a fixed polynomial differential system of degree $n \geq 2$.

Let the cubic system (1.2) have two parallel invariant straight lines $l_{1}$ and $l_{2}$. Then by a rotation of axes we can make them parallel to the axis of ordinates $(O y)$ and the linear part of (1.2) preserves the form. According to [6] the cubic system (1.2) has two invariant straight lines $l_{1}$ and $l_{2}$ parallel to the axis $O y$ if and only if the following coefficient conditions are satisfied

$$
a=f=k=p=r=0, \quad m\left(c^{2}-4 m\right) \neq 0 .
$$

In this case the cubic system (1.2) looks

$$
\begin{aligned}
& \dot{x}=y+c x y+m x^{2} y \equiv P(x, y), \\
& \dot{y}=-\left(x+g x^{2}+d x y+b y^{2}+s x^{3}+q x^{2} y+n x y^{2}+l y^{3}\right) \equiv Q(x, y) .
\end{aligned}
$$

The invariant straight lines $l_{1}$ and $l_{2}$ are

$$
l_{1,2} \equiv 2+\left(c \pm \sqrt{c^{2}-4 m}\right) x=0
$$

with the corresponding cofactors $K_{1,2}(x, y)=y\left(2 m x+c \pm \sqrt{c^{2}-4 m}\right) / 2$.

For cubic system (2.5) the problem of the existence of an invariant straight line was studied in [7], of an invariant conic in [6] and of an invariant cubic in [9].

\section{THE PROBLEM OF THE CENTER}

We consider the cubic differential system (2.5) with a singular point of a focus or a center at $O(0,0)$ having two parallel invariant straight lines of the form (2.6). The center conditions were obtained for system (2.5) when $m=l=0$ or $m=d=0$ in [12]; when $l=0$ in [14] and for a nine-parameter cubic system (2.5) that can be reduced to a Liénard type system in [3], [15].

As it was shown in [3], [15], the cubic system (2.5) by change of variables

$$
y=\frac{\left(1+g x+s x^{2}\right) Y}{1-\frac{d+q x}{3} Y}
$$


can be reduced to a Liénard type system of the form

$$
\begin{aligned}
& \dot{x}=Y\left(1+c_{1} x+c_{2} x^{2}+c_{3} x^{3}+c_{4} x^{4}\right), \\
& \dot{Y}=-x+\left(a_{0}+a_{1} x+a_{2} x^{2}+a_{3} x^{3}\right) Y^{2}+\left(b_{0}+b_{1} x+b_{2} x^{2}+b_{3} x^{3}+b_{4} x^{4}\right) Y^{3},
\end{aligned}
$$

where $a_{i}, b_{j}, c_{k}, i=\overline{0,3}, j=\overline{0,4}, k=\overline{1,4}$, are expressed in the coefficients of (2.5).

Using the method of generalized symmetry [1] and the Gröbner bases in [3], [15] there were obtained 14 sets of center conditions for system (3.8) ((2.5)) when $l \neq 0$ :

(i) $q=b d+d g-3 l,(d g-3 l+b d)(b d-3 l)+d^{2} s=0,9(b+2 g-c)(b d-3 l)+d(9 n+$ $\left.18 s-2 d^{2}\right)=0,\left(4 d^{2}+9(m-s)-9 g(c-g)\right)(b d-3 l)+d\left(2 d^{2} g+9 s(g-c)\right)=0 ;$

(ii) $q=b d+d g-3 l, m=(c-b-g)(b+g), n=2 b^{2}-2 b c+6 b g-2 c g+4 g^{2}-3 s$, $(c-2 g-b)(3 b+g)(b+g)+d l+(c-g+b) s=0,\left(b^{2}-b c+3 b g-c g+2 g^{2}-s\right)\left(b^{2}+\right.$ $b g+s)-l^{2}=0$;

(iii) $q=b d+d g-3 l, s=\left(3 b g-9 c g+2 d^{2}+12 g^{2}+6 m-6 n\right) / 18,12 d l-2 d^{2}(2 b+g)+$ $9(b+2 g-c)\left(g^{2}-4 s\right)=0,9 g^{2}(c-b-2 g)-2(b+2 g)\left(d^{2}-9 s\right)+3(2 d l+3 g n)=0$, $9(b+2 g-c)(b d-3 l)+d\left(9 n+18 s-2 d^{2}\right)=0 ;$

(iv) $q=b d+d g-3 l, s=-3(3 b+g)(b+g) / 4, c=(9 b+7 g) / 2, n=\left(417 b^{2}+374 b g+\right.$ $\left.12 d^{2}+57 g^{2}\right) / 64, m=\left(657 b^{2}+822 b g+12 d^{2}+265 g^{2}\right) / 64,(27 b+17 g)^{2}(3 b+g)+$ $4 d^{2}(17 b+11 g)=0,2(27 b+17 g) l+(5 b+3 g)(b+g) d=0 ;$

(v) $q=b d+d g-3 l, m=(c-9 b-7 g)(9 b-2 c+7 g), n=30 b^{2}-12 b c+44 b g+c^{2}-9 c g+16 g^{2}$ ， $s=(6 b+4 g-c)(c-6 b-5 g), d(5 b+4 g-c)(c-7 b-5 g)+l(7 c-45 b-33 g)=0$, $3 d^{2}-12(3 b+2 g)(11 b+10 g)+(9 b-c+7 g)(21 b+23 c+7 g)=0,2(b+g)(2 c-$ $11 g-15 b)(3 b+2 g)+(2 c-7 g-9 b)(c-6 g-8 b)(c-7 g-9 b)=0 ;$

(vi) $g=-b, m=n+3 s, q=-3 l, d=(b n+b s-c s) / l, 2 s^{2}+n s-l^{2}=0$;

(vii) $q=s=0, m=3(c-3 b-3 g)(b+g), n=2(3 b-c+3 g)(b+g), l=[(b+g) d] / 3$, $3(c-4 g-3 b)(3 b+2 g)+d^{2}=0$;

(viii) $l=-d(b+g) / 6, q=[3 d(b+g)] / 2, s=\left[3\left(-3 b^{2}-4 b g-g^{2}\right)\right] / 4,3(3 b+g)(c-g)+2 d^{2}=0$, $m=3(b+g)(2 c-3 b-3 g) / 4, n=(b+g)(6 b+3 g-c) / 2 ;$

(ix) $l=[d(c-2 g-2 b)] / 3, m=(2 c-3 g-3 b)(3 b+3 g-c), n=(c-2 g-3 b)(c-3 g-3 b)$, $q=d(3 b-c+3 g), s=-n, d^{2}+3(3 b+2 g-c)(c-g)=0$

(x) $c=3(b+g), s=-(3 b+2 g)(3 b+g), l=-(2 b+g) d, n=\left(48 b^{2}+44 b g-3 d^{2}+8 g^{2}\right) / 4$, $m=\left[3\left(-12 b^{2}-8 b g-d^{2}\right)\right] / 4, q=d(7 b+4 g),(7 b+5 g) d^{2}+4(3 b+2 g)^{2}(3 b+g)=0 ;$

(xi) $c=3(b+g), n=\left(2 d^{2}-9 b g-6 g^{2}-12 s\right) / 9, l=(d(2 b+g)) / 6, m=\left(18 b g-d^{2}+\right.$ $\left.12 g^{2}+6 s\right) / 6, q=(d g) / 2,36 b s-d^{2} g+24 g s=0 ;$

(xii) $q=b d+d g-3 l, m=3(2 c-3 b-3 g)(b+g) / 4, n=\left(2 d^{2}-9 b^{2}+6 b c-18 b g-3 g^{2}-12 s\right) / 6$, $3(2 c-3 b-5 g)[(3 b+g)(18 b+11 g)-4(3 b+2 g)(3 b+c-g)]+4 d^{2}(3 b-2 c+4 g)+12 s(6 b-$ $2 c+7 g)=0,3 d[(4 b+2 g)(3 b+c-g)-(3 b+g)(7 b+4 g)-4 s]+2 d^{3}+18 l(c-2 g)=0$, $2 d^{2}(2 c-b-3 g)+3(3 b+2 g)\left[(2 c-3 b-5 g)^{2}-4 s\right]-12 d l=0$;

(xiii) $q=b d+d g-3 l, m=3(c-3 b-3 g)(b+g), n=\left(d^{2}-9 b^{2}+3 b c-18 b g-6 g^{2}-6 s\right) / 3$, $d^{2}(2 c-6 b-7 g)-3 g(c-b-2 g)(2 c-b-2 g)+6 g(b+g)(5 b+3 g)+3 s(2 c-g)=0$, $9(3 b+c+g) l+3 d(2 b+g)(c-b-2 g)-3 d(b+g)(10 b+7 g)+d\left(d^{2}-6 s\right)=0$, $d^{2}(c-2 b-3 g)+3 g(b+g)(8 b+5 g)+3 g(b+c)(b-c+2 g)-3 d l+3 g s=0 ;$

(xiv) $q=b d+d g-3 l, m=(c-3 b-3 g)(3 b-2 c+3 g), n=\left(d^{2}-3(3 b-c+2 g)(3 b-\right.$ $2 c+4 g)-6 s) / 3,3 g(3 c-8 g-9 b)(2 c-4 g-3 b)-d^{2} g+3 s(6 c-13 g-12 b)=0$, $3 d\left(6 b^{2}+b g+2 s\right)+18 d(c-2 g-b)(c-2 g-3 b)-9 l(3 c-5 g-3 b)-d^{3}=0$, $9 d l-3 g(3 c-7 g-9 b)(2 c-4 g-3 b)-(3 b+g)\left(d^{2}-6 s\right)=0$. 
In Section 4 we will prove that in each of the cases (i) - (xiv) the cubic differential system (2.5) is Darboux integrable.

\section{DARBOUX INTEGRABILITY}

The problem of the center for polynomial differential systems is closely related to the problem of local integrability of the systems in some neighborhood of a singular point with purely imaginary eigenvalues. It is known from Poincaré and Lyapunov [1] that a singular point $O(0,0)$ is a center for system (2.5) if and only if the system has in some neighborhood of $O(0,0)$ a nonconstant analytic first integral

$$
F(x, y) \equiv x^{2}+y^{2}+\sum_{k=3}^{\infty} F_{k}(x, y)=C
$$

or an analytic integrating factor of the form

$$
\mu(x, y)=1+\sum_{k=1}^{\infty} \mu_{k}(x, y)
$$

where $F_{k}, \mu_{k}$ are homogeneous polynomials of degree $k$.

Definition 4.2. An integrating factor for system (2.5) on some open set $U$ of $\mathbb{R}^{2}$ is a $C^{1}$ function $\mu$ defined on $U$, not identically zero on $U$ such that

$$
P(x, y) \frac{\partial \mu}{\partial x}+Q(x, y) \frac{\partial \mu}{\partial y}+\mu\left(\frac{\partial P}{\partial x}+\frac{\partial Q}{\partial y}\right) \equiv 0 .
$$

We study the algebraic integrability of cubic differential system (2.5), called the Darboux integrability [4], [7]. It consists in constructing of a first integral or an integrating factor from the algebraic solutions of the form

$$
\Phi_{1}^{\alpha_{1}} \Phi_{2}^{\alpha_{2}} \cdots \Phi_{q}^{\alpha_{q}}
$$

where $\Phi_{j}=0, j=\overline{1, q}$ are invariant algebraic curves of (2.5) and $\Phi_{j} \in \mathbb{C}[x, y], \alpha_{j} \in \mathbb{C}$.

Theorem 4.1. The system (2.5) has a Darboux first integral (a Darboux integrating factor) of the form (4.12) if and only if there exists constants $\alpha_{j} \in \mathbb{C}$, not all identically zero such that

$$
\begin{gathered}
\alpha_{1} K_{1}(x, y)+\alpha_{2} K_{2}(x, y)+\cdots+\alpha_{q} K_{q}(x, y) \equiv 0, \\
\left(\sum_{j=1}^{q} \alpha_{j} K_{j}(x, y)+\frac{\partial P}{\partial x}+\frac{\partial Q}{\partial y} \equiv 0\right)
\end{gathered}
$$

where $K_{j}$ is the cofactor of $\Phi_{j}=0$ for $j=1, \ldots, q$.

The application of the method of Darboux to prove centers in all cases of quadratic differential systems was firstly proved in [16] and for cubic differential systems (1.2) with two invariant straight lines and one invariant conic was shown in [6]. Using Definition 2.1 for determining the invariant algebraic curves, the method of Darboux integrability and the identities (4.11), (4.14) we prove the following Theorem:

Theorem 4.2. Let $\operatorname{lm}\left(c^{2}-4 m\right) \neq 0$. The cubic differential system (2.5) is Darboux integrable in each of the cases (i)-(xiv).

Proof. In Case (i) the system (2.5) has three invariant straight lines:

$$
l_{1,2} \equiv 2+\left(c \pm \sqrt{c^{2}-4 m}\right) x=0, l_{3} \equiv 3 d+9 l x-3 b d x+d^{2} y=0
$$


and an integrating factor $\mu=l_{1}^{\alpha_{1}} l_{2}^{\alpha_{2}} l_{3}^{\alpha_{3}}$, where $u=\sqrt{c^{2}-4 m}$ and

$$
\begin{aligned}
& \alpha_{1}=\left[4 d^{4}+9 d^{2}\left(2 b^{2}+b c-b u-c u-u^{2}\right)+27 d l(c+3 u)-162 l^{2}\right] /\left[9 d^{2} u(c+u)\right], \\
& \alpha_{2}=\left(27 c d l-d^{2}\left(9 b^{2}+9 b c+2 d^{2}+9 m \alpha_{1}+18 m\right)+81 l^{2}\right) /\left(9 d^{2} m\right), \alpha_{3}=-3 .
\end{aligned}
$$

In Case (ii) the system (2.5) has four invariant straight lines:

$$
\begin{gathered}
l_{1} \equiv 1+(b+g) x=0, l_{2} \equiv 1+(c-b-g) x=0, \\
l_{3,4} \equiv\left(2 b+g \pm \sqrt{g^{2}-4 s}\right)\left(2+g x \pm x \sqrt{g^{2}-4 s}\right)+4 l y=0
\end{gathered}
$$

and an integrating factor $\mu=l_{1}^{\alpha_{1}} l_{2}^{\alpha_{2}} l_{3}^{\alpha_{3}} l_{4}^{\alpha_{4}}$, where $\alpha_{1}=1, \alpha_{3}=-\alpha_{4}-3$,

$$
\begin{aligned}
& \alpha_{2}=\left[l^{2}-g(b+g)\left(b^{2}+b g+s\right)-s\left(b^{2}+b g+s\right)^{3} l^{-2}\right] /\left[\left(b^{2}+b g+s\right)\left(g^{2}+b g-s\right)-l^{2}\right], \\
& \alpha_{4}=\left[l^{2}\left(g^{2}+b g-2 s-3(b+g) \sqrt{g^{2}-4 s}\right)-2 s\left(b^{2}+b g+s\right)^{2}\right] /\left[2 l^{2}(b+g) \sqrt{g^{2}-4 s}\right] .
\end{aligned}
$$

In Case (iii) the cubic system (2.5) has two invariant straight lines

$$
l_{1,2} \equiv 2+\left(c \pm \sqrt{c^{2}-4 m}\right) x=0
$$

and one invariant conic

$$
\begin{aligned}
\Phi_{2} & \equiv g\left(9 b g-9 c g+2 d^{2}+18 g^{2}\right)^{2} x^{2}+6 d g\left(9 b g-9 c g+2 d^{2}+18 g^{2}\right)(b+g) x y+ \\
& +12(3 g x+d y+3)\left(2 d^{2}(2 b-c+3 g)+9 g(b-c+2 g)^{2}\right)=0 .
\end{aligned}
$$

The system has an integrating factor $\mu=l_{1}^{\alpha_{1}} l_{2}^{\alpha_{2}} \Phi_{2}^{\alpha_{3}}$, where $u=\sqrt{c^{2}-4 m}$ and

$\alpha_{1}=\left[\alpha_{2}\left(u^{2}-c^{2}\right)+4 g(b+g)\right] /\left(c^{2}-u^{2}\right), \alpha_{3}=-3$,

$\alpha_{2}=\left(c^{2}-2 b c+2 b g+2 b u-3 c g-c u+2 g^{2}+3 g u\right) /[u(c-u)]$.

In Case (iv) the cubic system (2.5) has three invariant straight lines

$$
\begin{gathered}
l_{1,2} \equiv 4+(9 b+7 g \pm(5 b+3 g) \sqrt{(9 b+3 g) /(17 b+11 g)}) x=0, \\
l_{3} \equiv(5 b+3 g)(2+3(b+g) x)+4 l y=0
\end{gathered}
$$

and one invariant conic

$$
\begin{aligned}
\Phi_{2} & \equiv(17 b+11 g)\left[(3 b+g)(b+g)^{2} x^{2}+8 l(b+g) x y+\left(b^{2}-g^{2}\right) x+8 l y-2(b+g)\right]- \\
& -(5 b+3 g)^{2}(b+g)^{2} y^{2}=0 .
\end{aligned}
$$

The system has an integrating factor

$$
\mu=\frac{l_{3}^{2}}{l_{1}^{3 / 2} l_{2}^{3 / 2} \Phi_{2}^{5 / 2}} .
$$

In Case (v) the cubic system (2.5) has three invariant straight lines

$$
\begin{gathered}
l_{1} \equiv 1+(9 b-c+7 g) x=0, l_{2} \equiv 1-(9 b-2 c+7 g) x=0, \\
l_{3} \equiv(7 b-c+5 g)[1+(6 b-c+5 g) x]+l y=0
\end{gathered}
$$

and one invariant conic

$$
\begin{aligned}
\Phi_{2} & \equiv(5 b-c+4 g)\left[(6 b-c+4 g)(b+g) x^{2}+(5 b-c+3 g) x+\right. \\
& \left.+(8 b-c+6 g)(b+g) y^{2}-1\right]+2 l(b+g) x y+2 l y=0 .
\end{aligned}
$$

The system has an integrating factor

$$
\mu=\frac{l_{2} l_{3}^{2}}{l_{1}^{3 / 2} \Phi_{2}^{5 / 2}} .
$$

In Case (vi) the cubic system (2.5) has four invariant straight lines

$$
\begin{aligned}
& l_{1} \equiv 2+\left(c+\sqrt{c^{2}-4 m}\right) x=0, l_{3} \equiv b-\sqrt{b^{2}-4 s}-2 s x+2 l y=0, \\
& l_{2} \equiv 2+\left(c-\sqrt{c^{2}-4 m}\right) x=0, l_{4} \equiv b+\sqrt{b^{2}-4 s}-2 s x+2 l y=0
\end{aligned}
$$


and one invariant conic

$$
\Phi_{2} \equiv l s x^{2}-l^{2} x y-b l x-b s y-c s y+l=0 .
$$

The system has an integrating factor

$$
\mu=\frac{1}{l_{1} l_{2} l_{3} l_{4} \Phi_{2}} .
$$

In Case (vii) the cubic system (2.5) has two invariant straight lines

$$
l_{1} \equiv 1+3(b+g) x=0, l_{2} \equiv 1+(c-3 b-3 g) x=0
$$

and one invariant cubic

$$
\Phi_{3} \equiv(b+g) d^{3} y^{3}+3(3 b+2 g)\left(3(1+g x)^{2}+3 d(1+g x) y+d^{2} y^{2}\right)=0 .
$$

The system has an integrating factor

$$
\mu=\frac{1}{l_{1}^{1 / 3} l_{2} \Phi_{3}} .
$$

In Case (viii) the cubic system (2.5) has two invariant straight lines

$$
l_{1} \equiv 2+(2 c-3 b-3 g) x=0, l_{2} \equiv 2+3(b+g) x=0
$$

and one invariant cubic

$$
\begin{aligned}
\Phi_{3} & \equiv(b+g)(9 b x+3 g x-2 d y)^{3}-6(3 b+g)\left[3(3 b+g)(3 b+5 g) x^{2}-\right. \\
& \left.-12 d g x y-4 d^{2} y^{2}+6(3 b-g) x-12 d y-12\right]=0 .
\end{aligned}
$$

The system has an integrating factor

$$
\mu=\frac{1}{l_{1} l_{2}^{2 / 3} \Phi_{3}} .
$$

In Case (ix) the cubic system (2.5) has two invariant straight lines

$$
l_{1} \equiv 1+(2 c-3 b-3 g) x=0, l_{2} \equiv 1+(3 b+3 g-c) x=0
$$

and one invariant cubic

$$
\begin{aligned}
\Phi_{3} & \equiv(c-3 b-2 g)\left[3(3 b-c+3 g)(3 b-c+2 g) x^{3}+3 d(c-3 b-3 g) x^{2} y+\right. \\
& \left.+3(c-g)(c-3 b-3 g) x y^{2}+d(2 b+2 g-c) y^{3}\right]+3(3 b+2 g-c)(c-g) y^{2}- \\
& -3 d g x y+3(3 b-c+2 g)(3 b-c+4 g) x^{2}+3(3 b+g-c) x-3 d y-3=0 .
\end{aligned}
$$

The system has an integrating factor

$$
\mu=\frac{1}{l_{1}^{1 / 3} l_{2}^{2 / 3} \Phi_{3}} .
$$

In Case (x) the cubic system (2.5) has three invariant straight lines

$$
l_{1,2} \equiv 2+\left(3 b+3 g \pm \sqrt{45 b^{2}+42 b g+3 d^{2}+9 g^{2}}\right) x=0, l_{3} \equiv 2+2(3 b+2 g) x-d y=0
$$

and one invariant cubic

$$
\begin{aligned}
\Phi_{3} & \equiv 2(3 b+2 g)(3 b+g)^{2} x^{3}-3 d(5 b+3 g)(3 b+g) x^{2} y+6 d^{2}(2 b+g) x y^{2}+ \\
& +4 d(2 b+g)^{2} y^{3}+6 d(b+g) x y-6(b+g)(3 b+g) x^{2}-6 b x+3 d y+2=0 .
\end{aligned}
$$

The system has an integrating factor

$$
\mu=\frac{1}{l_{1}^{2 / 3} l_{2}^{2 / 3} l_{3}^{1 / 2} \Phi_{3}^{5 / 6}} .
$$


In Case (xi) the cubic system (2.5) has two invariant straight lines

$$
l_{1,2} \equiv 6+\left[9(b+g) \pm \sqrt{3\left(27 b^{2}+18 b g+2 d^{2}+3 g^{2}-12 s\right)}\right] x=0
$$

and one invariant quartic

$$
\begin{aligned}
\Phi_{4} & \equiv x(6 b y+d x+4 g y)^{3} d g^{2}+4(3 b+2 g)\left[6 d^{2} g^{2} x^{3}+3 d g\left(18 b g+d^{2}+12 g^{2}\right) x^{2} y+\right. \\
& +6 g\left(18 b g+d^{2}+12 g^{2}\right) x^{2}+2 d\left(2 b d^{2}+3 b g^{2}+d^{2} g+2 g^{3}\right) y^{3}+ \\
& \left.+6(3 b+2 g)\left(2 d^{2} g x y^{2}+9 d g x y+12 g x+2 d^{2} y^{2}+6 d y+6\right)\right]=0 .
\end{aligned}
$$

The system has an integrating factor

$$
\mu=\frac{1}{l_{1}^{1 / 3} l_{2}^{1 / 3} \Phi_{4}} .
$$

In Case (xii) the cubic system (2.5) has two invariant straight lines

$$
l_{1} \equiv 2+3(b+g) x=0, l_{2} \equiv 2+(2 c-3 b-3 g) x=0
$$

and one invariant quartic

$$
\begin{aligned}
\Phi_{4} & \equiv 3 s\left(a_{20}-g^{2}-s\right) x^{4}+3 a_{31} x^{3} y+3 a_{22} x^{2} y^{2}+\left(9 b a_{03}+6 g a_{03}-d^{2} l\right) x y^{3}+ \\
& +3 g\left(a_{20}-g^{2}\right) x^{3}+3\left(d a_{20}+b d g-d s-3 g l\right) x^{2} y+2 d(b d+d g-3 l) x y^{2}+ \\
& +3 a_{03} y^{3}+3 a_{20} x^{2}+3(b d+2 d g-3 l) x y+d^{2} y^{2}+6 g x+3 d y+3=0,
\end{aligned}
$$

where

$$
\begin{aligned}
& a_{03}=\left(b^{2} d+2 b d g+6 b l-6 c l+d g^{2}+12 g l\right) / 6 \\
& a_{31}=(b d+d g-3 l) a_{20}-b d g^{2}-b d s-d g^{3}-d g s+3 g^{2} l+3 l s, \\
& a_{22}=\left(a_{20} d^{2}+b^{2} d^{2}+2 b d^{2} g-6 b d l-2 d^{2} s-6 d g l+9 l^{2}\right) / 3, \\
& a_{20}=\left(27 b^{2} g-18 b c g+8 b d^{2}+54 b g^{2}+6 c g^{2}+4 d^{2} g-24 d l-3 g^{3}\right) /[6(2 c-3 g)] .
\end{aligned}
$$

The system has an integrating factor

$$
\mu=\frac{l_{1}^{1 / 3}}{l_{2} \Phi_{4}} .
$$

In Case (xiii) the cubic system (2.5) has two invariant straight lines

$$
l_{1} \equiv 1+3(b+g) x=0, l_{2} \equiv 1+(c-3 b-3 g) x=0
$$

and one invariant quartic

$$
\begin{aligned}
\Phi_{4} & \equiv 3 s\left(a_{20}-g^{2}-s\right) x^{4}+3 a_{31} x^{3} y+3 a_{22} x^{2} y^{2}+\left(9 b a_{03}+6 g a_{03}-d^{2} l\right) x y^{3}+ \\
& +3 g\left(a_{20}-g^{2}\right) x^{3}+3\left(d a_{20}+b d g-d s-3 g l\right) x^{2} y+2 d(b d+d g-3 l) x y^{2}+ \\
& +3 a_{03} y^{3}+3 a_{20} x^{2}+3(b d+2 d g-3 l) x y+d^{2} y^{2}+6 g x+3 d y+3=0,
\end{aligned}
$$

where

$$
\begin{aligned}
& a_{03}=\left(2 b^{2} d+4 b d g+3 b l-3 c l+2 d g^{2}+6 g l\right) / 3, \\
& a_{31}=(b d+d g-3 l) a_{20}-b d g^{2}-b d s-d g^{3}-d g s+3 g^{2} l+3 l s, \\
& a_{22}=\left(a_{20} d^{2}+b^{2} d^{2}+2 b d^{2} g-6 b d l-2 d^{2} s-6 d g l+9 l^{2}\right) / 3, \\
& a_{20}=\left(54 b^{2} g-18 b c g+4 b d^{2}+108 b g^{2}-6 c g^{2}+2 d^{2} g-12 d l+39 g^{3}\right) /[3(2 c-3 g)] .
\end{aligned}
$$

The system has an integrating factor

$$
\mu=\frac{1}{l_{1}^{1 / 3} l_{2} \Phi_{4}} .
$$

In Case (xiv) the cubic system (2.5) has two invariant straight lines

$$
l_{1} \equiv 1+(3 b-c+3 g) x=0, l_{2} \equiv 1+(2 c-3 b-3 g) x=0
$$


and one invariant quartic

$$
\begin{aligned}
\Phi_{4} & \equiv 3 s\left(a_{20}-g^{2}-s\right) x^{4}+3 a_{31} x^{3} y+3 a_{22} x^{2} y^{2}+\left(9 b a_{03}+6 g a_{03}-d^{2} l\right) x y^{3}+ \\
& +3 g\left(a_{20}-g^{2}\right) x^{3}+3\left(d a_{20}+b d g-d s-3 g l\right) x^{2} y+2 d(b d+d g-3 l) x y^{2}+ \\
& +3 a_{03} y^{3}+3 a_{20} x^{2}+3(b d+2 d g-3 l) x y+d^{2} y^{2}+6 g x+3 d y+3=0,
\end{aligned}
$$

where

$$
\begin{aligned}
& a_{03}=\left(\left(8(b+g)(b+g-c)+2 c^{2}\right) d+l(3 b-3 c+6 g)\right) / 3 \\
& a_{31}=(b d+d g-3 l) a_{20}-b d g^{2}-b d s-d g^{3}-d g s+3 g^{2} l+3 l s, \\
& a_{22}=\left(a_{20} d^{2}+b^{2} d^{2}+2 b d^{2} g-6 b d l-2 d^{2} s-6 d g l+9 l^{2}\right) / 3 \\
& a_{20}=\left[54 b g(b-c+2 g)+2 d^{2}(2 b+g)+12 c^{2} g-42 c g^{2}-12 d l+39 g^{3}\right] /[3(2 c-3 g)] .
\end{aligned}
$$

The system has an integrating factor

$$
\mu=\frac{l_{1}^{1 / 3}}{l_{2}^{1 / 3} \Phi_{4}} .
$$

Note that the integrability conditions (i), (ii), (vi), (x) were obtained in [7] and the integrability conditions (iii), (iv), (v) were determined in [6].

\section{MAIN RESULTS}

The application of the method of Darboux to prove centers in all cases (i) - (xiv) of cubic differential systems (2.5) was firstly shown in this paper.

Theorem 5.3. The cubic differential system $\left\{(2.5), \operatorname{lm}\left(c^{2}-4 m\right) \neq 0\right\}$ has a center at $O(0,0)$ if and only if it is Darboux integrable.

The proof of the main result, Theorem 5.3, follows directly from Theorem 4.2 and the center conditions (i) - (xiv).

\section{REFERENCES}

[1] Amel'kin, V. V.; Lukashevich, N. A.; Sadovskii, A. P. Non-linear oscillations in the systems of second order. Belarusian University Press, Minsk 1982 (in Russian).

[2] Arcet, B.; Romanovski, V. G. On some reversible cubic systems. Mathematics 9 (2021), 1446.

[3] Bondar, Y. L.; Sadovskii, A. P. Variety of the center and limit cycles of a cubic system, which is reduced to Lienard form. Bul. Acad. de Ştiinţe a Moldovei. Matematica 46 (2004), 71-90.

[4] Christopher, C.; Llibre, J.; Pantazi, C.; Zhang, X. Darboux integrability and invariant algebraic curves for planar polynomial systems. J. Phys. A. 35 (2002), no. 10, 2457-2476.

[5] Cozma, D. Şubă, A. The solution of the problem of center for cubic differential systems with four invariant straight lines. Sci. Annals of the "Al. I. Cuza" University, Mathematics XLIV (1998), 517-530.

[6] Cozma, D. The problem of the center for cubic systems with two parallel invariant straight lines and one invariant conic. Nonlinear Differ. Equ. and Appl. 16 (2009), 213-234.

[7] Cozma, D. Integrability of cubic systems with invariant straight lines and invariant conics. Ştiinţa, Chişinău, 2013.

[8] Cozma, D. Darboux integrability and rational reversibility in cubic systems with two invariant straight lines. Electron. J. Differ. Equ. 2013 (2013), no. 23, 1-19.

[9] Cozma, D. The problem of the center for cubic systems with two parallel invariant straight lines and one invariant cubic. ROMAI Journal 11 (2015), 63-75.

[10] Cozma, D.; Dascalescu, A. Integrability conditions for a class of cubic differential systems with a bundle of two invariant straight lines and one invariant cubic. Bul. Acad. de Şt. a Republicii Moldova. Matematica 86 (2018), 120-138.

[11] Dukarić, M. On integrability and cyclicity of cubic systems. Electr. J. Qual. Theory Differ. Equ. 55 (2020), 1-19.

[12] Hill, J. M., Lloyd, N. G., Pearson, J. M. Centres and limit cycles for an extended Kukles system. Electronic Journal of Diff. Equations 2007 (2007), no. 119, 1-23.

[13] Prelle, M. J.; Singer, M. F. Elementary first integrals of differential equations. Trans. Amer. Math. Soc. 273 (1983), 215-229. 
[14] Sadovskii, A. P. Center conditions and limit cycles in a cubic system of differential equations. Diff. Equations 36 (2000), 113-119.

[15] Sadovskii, A. P.; Scheglova, T. V. Solution of the center-focus problem for a nine-parameter cubic system Diff. Equations 47 (2011), 208-223.

[16] Schlomiuk, D. Algebraic and geometric aspects of the theory of polynomial vector fields. in Bifurcations and periodic orbits of vector fields (D.Schlomiuk, Ed.), Kluwer Academic Publishes, 1993 pp. 429-467

[17] Şubă, A.; Cozma, D. Solution of the problem of center for cubic differential systems with three invariant straight lines in generic position. Qual. Theory Dyn. Syst. 6 (2005), 45-58.

Department of Mathematical ANALYsis AND DifFerential EQuations

TIRASPOL STATE UNIVERSITY

5 GH. IAblociKin, MD 2063, Moldova

Email address: dcozmaegmail.com 